\title{
The Benefits of Micronized Purified Flavonoid Fraction (MPFF) Throughout the Progression of Chronic Venous Disease
}

Andrew N. Nicolaides

Received: November 26, 2019 / Published online: January 22, 2020

(c) The Author(s) 2020

\section{ABSTRACT}

At the 2019 European Venous Forum in Zurich Switzerland, a symposium entitled "State of the art: benefits of MPFF throughout CVD progression" was held to discuss the developing treatment strategies for patients at all stages of chronic venous disease (CVD). At the early stages of CVD, management should be focused on preventing disease progression through lifestyle changes and conservative treatment; treatment can also include venoactive drugs (VAD) such as micronized purified flavonoid fraction (MPFF; Daflon ${ }^{\circledR}$ ), which is the most well-known and most widely prescribed VAD in Europe. As the disease progresses, patients who require interventional procedures (e.g., endovenous procedure or sclerotherapy) can also benefit from MPFF treatment in the recovery period after the procedure, as MPFF has been shown to reduce periprocedural pain and bleeding (hematoma), and to improve CVD symptoms during this period. Management of CVD in patients with venous leg ulcers (VLU) is the most challenging; in these patients,

Enhanced Digital Features To view enhanced digital features for this article go to https://doi.org/10.6084/ m9.figshare.11417802.

\section{A. N. Nicolaides $(\square)$}

Department of Surgery, University of Nicosia

Medical School, Nicosia, Cyprus

e-mail: anicolai@cytanet.com.cy recommended adjunct therapies to be combined with standard compression therapy include VAD (MPFF) and non-VAD drugs (pentoxifylline and sulodexide) which have been shown to speed VLU healing in comparison with compression therapy alone.

Keywords: Chronic venous disease; Chronic venous reflux; Endovenous ablation; Sclerotherapy; Venoactive drugs; Venous leg ulcers

\section{Key Summary Points}

At the 2019 European Venous Forum in Zurich Switzerland, a symposium entitled "State of the art: benefits of MPFF throughout CVD progression" was held to discuss the developing treatment strategies for patients at all stages of chronic venous disease (CVD).

At the early stages of CVD, management should be focused on preventing disease progression through lifestyle changes and conservative treatment; treatment can also include venoactive drugs (VAD) such as micronized purified flavonoid fraction (MPFF; Daflon ${ }^{\circledR}$ ), which is the most wellknown and most widely prescribed VAD in Europe. 
As the disease progresses, patients who require interventional procedures (e.g., endovenous procedure or sclerotherapy) can also benefit from MPFF treatment in the recovery period after the procedure, as MPFF has been shown to reduce periprocedural pain and bleeding (hematoma), and to improve CVD symptoms during this period.

Management of CVD in patients with venous leg ulcers (VLU) is the most challenging; in these patients, recommended adjunct therapies to be combined with standard compression therapy include VAD (MPFF) and nonVAD drugs (pentoxifylline and sulodexide) which have been shown to speed VLU healing in comparison with compression therapy alone.

\section{INTRODUCTION}

Chronic venous disease (CVD) is a common and progressive disorder of the legs. Though etiologies may differ, the principal factors that lead to its development are chronic venous reflux and venous hypertension [1]. These conditions precipitate a cascade of pathological and inflammatory responses that increase over time, including endothelial activation, leukocyte adhesion, deterioration of the vein walls, increases in capillary permeability, and leukocyte and reticulocyte infiltration with release of proinflammatory mediators and growth factors $[2,3]$. Persistent vein dilatation, inappropriate cell proliferation in the vein walls, and changes in collagen synthesis lead to the development of unsightly varicose veins, while the resulting edema and chronic inflammation cause symptoms of pain, itching, cramps, and feelings of leg heaviness. Patients with such manifestations of CVD seek medical treatment for symptom relief and removal of varicose veins. As the disease progresses further, patients may develop changes and hardening of the skin due to capillary leakage and accumulation of extracellular proteins. Such changes place patients at high risk of developing venous leg ulcers (VLU), which heal slowly and are prone to recurrence. VLU impose heavy burdens on healthcare systems and on patient quality of life (QoL).

At the 20th annual meeting of the European Venous Forum (EVF; June 27-29, 2019, Zurich, Switzerland), a symposium entitled "State of the art: benefits of MPFF throughout CVD progression" sponsored by Servier (France) was held to discuss developing treatment strategies for patients at all stages of CVD. The symposium covered treatments for early stage symptomatic CVD (CEAP classes COs-C2), more advanced stages of CVD with varicose veins and chronic edema (C2-C4), and the most severe stages involving VLU (C5-C6). The symposium panel included three experts in CVD research and treatment: Professor Armando Mansilha from the Faculdade de Medicina da Universidade in Porto, Portugal; Dr. Fedor Lurie from the JOBST Vascular Institute in Toledo, Ohio; and Professor Andrew Nicolaides from the University of Nicosia Medical School in Nicosia, Cyprus.

CVD is common throughout the world, but its prevalence is highest in Western countries [4]. In European countries, CVD care may consume up to $2 \%$ of national healthcare budgets [5]. Unfortunately, as we have little or no means of preventing the disease, CVD will continue to increase in prevalence along with the growing population of older adults and the increasing prevalence of obesity. Indeed, increasing age and obesity are two of the primary risk factors for CVD. Others include sedentary occupations and lifestyles, genetic factors, pregnancy, and previous damage to the legs (e.g., deep vein thrombosis). Once CVD develops, it has a high probability of progressing. In the classic example of the Edinburgh Vein Study, progression occurred in more than $57 \%$ of the patients with varicose veins or chronic venous insufficiency (CVI; class C3 or greater) over 13 years of followup [3].

It is not yet possible to predict which individuals will develop CVD, making effective prevention strategies difficult to achieve. On the other hand, effective treatment strategies now exist for patients at all stages of CVD. Overall treatment objectives are to relieve 
symptoms, reduce visible signs, prevent progression to greater severity, and improve QoL. Ideally, interventions should reduce or eliminate the need for further treatment.

For patients with early stage CVD, lifestyle changes should be recommended, if necessary, to reduce the impact of risk factors such as obesity and inactivity. Patients can also be treated with venoactive drugs (VAD) and with outpatient procedures such as sclerotherapy or endovenous ablation (EVA) techniques to address telangiectasia (spider veins) and varicose veins in situ. Compression therapy and surgery may also be used. For VAD treatment, micronized purified flavonoid fraction (MPFF; Daflon $^{\circledR}$ ) is the most well-known and most widely prescribed VAD in Europe, though it is not yet available in the USA. MPFF has pleiotropic pharmacological effects, which act at various levels of pathological dysfunction in patients with CVD. MPFF has anti-inflammatory activities, reduces endothelial cell activation and leukocyte adhesion, and increases capillary resistance and integrity [4]. As a result, MPFF treatment produces clinical benefits in patients with all classes of CVD. It reduces CVD signs and symptoms such as pain, heaviness, edema, and skin changes. It also improves venous tone, QoL, and promotes ulcer healing $[3,6,7]$.

MPFF treatment in symptomatic women without varicose veins (COs) reduced the frequency of evening (after work) reflux and associated symptoms by $85 \%$ after 2 months [8]. In women with symptoms and early signs (telangiectasia, C1s), 3 months of MPFF treatment improved or eliminated leg heaviness, fatigue, pain, and night cramps in the majority of patients [9].

As the disease progresses, patients who require interventions can also benefit from MPFF treatment in the post-procedural recovery period. Two studies reported that in patients treated with MPFF before an endovenous procedure or sclerotherapy and for several days afterward, venous clinical severity scores and CVD-associated symptoms improved to significantly greater degrees than in patients who had the procedures without MPFF treatment $[10,11]$. To investigate this aspect of VAD treatment in greater detail, Mansilha et al. conducted a systematic review of the clinical evidence for the effects of VAD treatment after surgical or endovenous intervention [12]. Unfortunately, few studies qualified for the analysis but among those retained, the majority of studies found significant benefits in pain relief, reduced bleeding (hematoma), and CVD symptom improvement.

Recent studies have proposed that perioperative inflammation, which correlated with postprocedural pain in HLS and RFA, may be a key factor in patient discomfort as well as in varicose vein recurrence $[13,14]$. The possibility that efforts to reduce perioperative inflammation may also reduce recurrence in the longer term is an attractive hypothesis. VAD, such as MPFF, not only reduce inflammation but provide other CVD-specific benefits to patients undergoing HLS or EVA [11, 12, 15, 16]. The long-term benefits of post-procedural VAD treatment await investigation in a well-designed, placebo-controlled randomized clinical trial.

VLU develop as a consequence of chronic leg edema, inflammation, skin capillary breakdown, and lymphatic damage. Management of these patients is the most challenging and, indeed, the most costly among patients with CVD. These lesions heal slowly, even with appropriate treatment, and have high rates of recurrence. For many patients that develop severe CVD, VLU impose a heavy healthcare burden on them and their caregivers, and reduce QoL. The socioeconomic impact of VLU is substantial and can account for up to $1-2 \%$ of national healthcare budgets [17-19].

The objectives of medical management of VLU are to speed healing and prevent recurrence. Management should begin with a careful assessment to identify the incompetent veins responsible for the condition. Dressings and compression are standard initial treatment, whereas surgery may also be needed to remove incompetent veins [7]. Intermittent pneumatic compression may also be beneficial when used with standard compression [20].

VAD therapy along with compression can speed VLU healing compared with compression alone $[7,21]$. In a meta-analysis of clinical trials comparing standard compression therapy with or without adjunct systemic treatment with 
MPFF, VLU healing probabilities were 32\% higher with MPFF and healing time was shortened by 4 weeks [21]. Two other non-VAD drugs, pentoxifylline and sulodexide, have also shown efficacy in improving VLU healing. In the current European CVD management guidelines (2018), MPFF, pentoxifylline, and sulodexide are recommended adjunct therapies to be combined with standard compression therapy [10].

\section{CONCLUSIONS}

CVD at each stage of severity presents specific healthcare challenges to patients and physicians. At the early stages, management should be focused on preventing disease progression through lifestyle changes and conservative treatment. As CVD progresses, surgical and outpatient procedures to remove or ablate varicose veins and eliminate venous reflux are the standard of care. In the most severe forms, standard VLU treatment includes compression bandaging, but surgery may also be necessary. Indeed, various management options exist for patients at each stage and treatment strategies will depend on the characteristics of individual patients.

However, it is also clear from controlled clinical trials and multiple meta-analyses that VAD such as MPFF provide therapeutic benefits at each stage of CVD, by reducing CVD-specific symptoms and inflammation, periprocedural pain, and by speeding healing of VLU. We hope that physicians concerned about providing optimal care for their patients with CVD will find the accompanying summaries from this EVF symposium to be informative starting points to improve their understanding of the challenges of managing patients with CVD effectively and the treatment strategies available to them.

\section{ACKNOWLEDGEMENTS}

Funding. This supplement has been sponsored by Servier. Article processing charges and the open access fee were funded by Servier.
Medical writing. Medical writing services were provided by Dr. Kurt Liittschwager (4Clinics, France) and were funded by Servier.

Authorship. Dr. Andrew N. Nicolaides meets the International Committee of Medical Journal Editors (ICMJE) criteria for authorship for this article, takes responsibility for the integrity of the work as a whole, and has approved this version for publication.

Prior presentation. This article and all of the articles in this supplement are based on the international satellite symposium at the European Venous Forum (June 2019, Zurich, Switzerland).

Disclosures. Dr. Andrew N. Nicolaides declares having received speaker honoraria from Medtronic, Servier, Pierre Fabre, and Alfasigma.

Compliance with Ethics Guidelines. This article is based on previously conducted studies and does not contain any studies with human participants or animals performed by the author.

Open Access. This article is licensed under a Creative Commons Attribution-NonCommercial 4.0 International License, which permits any non-commercial use, sharing, adaptation, distribution and reproduction in any medium or format, as long as you give appropriate credit to the original author(s) and the source, provide a link to the Creative Commons licence, and indicate if changes were made. The images or other third party material in this article are included in the article's Creative Commons licence, unless indicated otherwise in a credit line to the material. If material is not included in the article's Creative Commons licence and your intended use is not permitted by statutory regulation or exceeds the permitted use, you will need to obtain permission directly from the copyright holder. To view a copy of this licence, visit http://creativecommons.org/licenses/by$\mathrm{nc} / 4.0 /$. 


\section{REFERENCES}

1. Bergan JJ, Schmid-Schonbein GW, Smith PD, Nicolaides AN, Boisseau MR, Eklof B. Chronic venous disease. N Engl J Med. 2006;355(5):488-98.

2. Bergan J. Molecular mechanisms in chronic venous insufficiency. Ann Vasc Surg. 2007;21(3):260-6.

3. Mansilha A, Sousa J. Pathophysiological mechanisms of chronic venous disease and implications for venoactive drug therapy. Int $\mathrm{J}$ Mol Sci. 2018;19(6).

4. Rabe E, Guex JJ, Puskas A, Scuderi A, Fernandez Quesada F. Epidemiology of chronic venous disorders in geographically diverse populations: results from the Vein Consult Program. Int Angiol. 2012;31(2):105-15.

5. Laing W. Chronic venous diseases of the leg. London: Office of Health Economics; 1992.

6. Kakkos SK, Nicolaides AN. Efficacy of micronized purified flavonoid fraction (Daflon ${ }^{\circledR}$ ) on improving individual symptoms, signs and quality of life in patients with chronic venous disease: a systematic review and meta-analysis of randomized doubleblind placebo-controlled trials. Int Angiol. 2018;37(2):143-54.

7. Nicolaides A, Kakkos S, Baekgaard N, et al. Management of chronic venous disorders of the lower limbs. Guidelines according to scientific evidence. Part I. Int Angiol. 2018;37(3):181-254.

8. Tsoukanov YT, Tsoukanov AY. Great saphenous vein transitory reflux in patients with symptoms related to chronic venous disorders, but without visible signs (COs), and its correction with MPFF treatment. Phlebolymphology. 2017;22(1):3-11.

9. Tsukanov YT, Nikolaichuk AI. Orthostatic-loadinginduced transient venous refluxes (day orthostatic loading test), and remedial effect of micronized purified flavonoid fraction in patients with telangiectasia and reticular vein. Int Angiol. 2017;36(2): 189-96.

10. Bogachev VY, Boldin BV, Turkin PY. Administration of micronized purified flavonoid fraction during sclerotherapy of reticular veins and telangiectasias: results of the National, Multicenter, Observational Program VEIN ACT PROLONGEDC1. Adv Ther. 2018;35(7):1001-8.

11. Bogachev VY, Golovanova OV, Kuzhetsov AN, Shekoian AO, The DECISION Investigators. Can micronized purified flavonoid fraction* (MPFF) improve outcomes of lower extremity varicose vein endovenous treatment? First results from the DECISION study. Phlebolymphology. 2013;20(4): $181-7$.

12. Mansilha A, Sousa J. Benefits of venoactive drug therapy in surgical or endovenous treatment for varicose veins: a systematic review. Int Angiol 2019;38(4):291-8.

13. Taengsakul N, Saikaew $\mathrm{T}$, Chaiaroon $\mathrm{N}$, et al. Inflammatory responses in varicose veins surgery: conventional venous stripping and endovenous radiofrequency ablation (EV-RFA). J Vasc Endovascr Ther 2019;4(1):9.

14. Ashpitel HF, Dabbs EB, Salguero FJ, Nemchand JL, La Ragione RM, Whiteley MS. Histopathologic differences in the endovenous laser ablation between jacketed and radial fibers, in an ex vivo dominant extrafascial tributary of the great saphenous vein in an in vitro model, using histology and immunohistochemistry. J Vasc Surg Venous Lymphat Disord. $2019 ; 7(2): 234-45$.

15. Veverkova L, Kalac J, Jedlicka V, Wechsler J. Analysis of surgical procedures on the vena saphena magna in the Czech Republic and an effect of Detralex during its stripping. Rozhl Chir. 2005;84(8):410-2 (4-6).

16. Veverková L, Kalač J, Jedlička V, Wechsler J. Analysis of the various procedures used in great saphenous vein surgery in the Czech Republic and benefit of Daflon $500 \mathrm{mg}$ to postoperative symptoms. Phlebolymphology. 2006;13(4):193-9.

17. O'Donnell TF Jr, Balk EM. The need for an Intersociety Consensus Guideline for venous ulcer. J Vasc Surg. 2011;54(6):83S-90S.

18. O'Donnell TF Jr, Passman MA. Clinical practice guidelines of the Society for Vascular Surgery (SVS) and the American Venous Forum (AVF)—management of venous leg ulcers. Introduction. J Vasc Surg. 2014;60(2 Suppl):1S-2S.

19. Ruckley CV. Socioeconomic impact of chronic venous insufficiency and leg ulcers. Angiology. 1997;48(1):67-9.

20. Nelson EA, Hillman A, Thomas K. Intermittent pneumatic compression for treating venous leg ulcers. Cochrane Database Syst Rev. 2014;5: CD001899.

21. Coleridge-Smith P, Lok C, Ramelet AA. Venous leg ulcer: a meta-analysis of adjunctive therapy with micronized purified flavonoid fraction. Eur J Vasc Endovasc Surg. 2005;30(2):198-208. 